\title{
Agenda culinaria para 1896 de Blanco Prieto. Un estudio histórico del léxico de la gastronomía
}

\author{
Agenda culinaria para 1896 by Blanco Prieto. \\ A historical study of the lexicon of gastronomy
}

\author{
Francesca Dalle Pezze \\ francesca.dallepezze@univr.it \\ Elisa SARTOR \\ elisa.sartor@univr.it
}

Università degli Studi di Verona

\begin{abstract}
Resumen: El objetivo de esta contribución es dúplice: en primer lugar, presentar Agenda culinaria para 1896. Libro de la compra. Con minutas y recetas para cada uno de los días del año, un libro que muestra características editoriales que hacen que no sea solo un recetario, sino también un producto de consumo que se enmarca en el auge de las publicaciones relacionadas con la gastronomía a finales del siglo XIX; en segundo lugar, ofrecer un estudio del léxico culinario contenido en esta obra, con el fin de detectar extranjerismos, neologismos y otras creaciones léxicas surgidas a raíz del contacto entre la cocina española e ingredientes y platos procedentes de otros países y culturas. Así pues, se ha analizado la trayectoria lexicográfica de tres términos (kari, uspot, pilaw) que ofrecen una muestra de la circulación europea e internacional del léxico de la comida y la gastronomía, cada uno mediante vías de penetración y acomodación en el caudal léxico español diferentes, como la doble entrada (karr) o el préstamo efímero (uspot); asimismo, se ha podido establecer un caso de retrodatación (pilaw).
\end{abstract}

Palabras clave: galicismos. gastronomía, recetarios, lexicología, lexicografía, préstamo.

\begin{abstract}
The objective of this paper is two-fold: firstly, it aims at presenting Agenda culinaria para 1896. Libro de la compra. Con minutas y recetas para cada uno de los días del año, a book that displays editorial features that turn it into something more than a recipe book. As a matter of fact, it may be considered a consumer product that fits into the boom in publications related to cookery in the late 19th century. Secondly, it offers a study of the gastronomy lexicon that can be found in this volume, with the purpose of detecting foreign words, neologism and other lexical creations brought about by the contact between Spanish cuisine and ingredients and those from other countries and cultures. Therefore, this paper analyses the lexicographical trajectory of three terms (kari, uspot, pilaw) that offer a sample of the European and international circulation of lexicon of food and cookery, each one showing different paths and strategies of entry in the Spanish lexicon: double entry (kari), momentary loanwords (uspot), and predating (pilaw).
\end{abstract}

Keywords: gastronomy, recipe books, lexicology, lexicography, loanword.

\footnotetext{
* Este estudio se enmarca en la actividad del grupo de investigación El léxico del español en su historia (LEHist) de la Universidad de Verona (https://www.dlls.univr.it/?ent=grupporic\&id=181). Aunque el artículo ha sido elaborado conjuntamente, a efectos académicos Francesca Dalle Pezze es la autora de los apartados 1 y 2 y Elisa Sartor de los apartados 3 y 4 .
}

DATA PRESENTACIÓ: 14/11/2021 ACCEPTACIÓ: 30/11/2021 · PUBLICACIÓ: 24/12/2021

SCRIPTA, Revista internacional de literatura i cultura medieval i moderna, núm. 18 / desembre 2021 / pp. 115-127 ISSN: 2340-4841 · doi:10.7203/SCRIPTA.18.22767 
Francesca Dalle Pezze \& Elisa Sartor. Agenda culinaria para 1896 de Blanco Prieto. Un estudio histórico del léxico de la gastronomía

\section{Introducción}

Esta contribución pretende ahondar en el estudio histórico del léxico mediante el análisis de una obra del ámbito de la gastronomía escasamente investigada hasta ahora. El objetivo de este artículo es dúplice: en primer lugar, se presentará Agenda culinaria para 1896. Libro de la compra. Con minutas y recetas para cada uno de los días del año, un libro que, como veremos, muestra características editoriales que hacen que no sea solo un recetario, sino también un producto de consumo que se enmarca en el auge de las publicaciones relacionadas con la gastronomía a finales del siglo XIX; en segundo lugar, se ofrecerá un estudio del léxico culinario contenido en esta obra, con el fin de detectar extranjerismos, neologismos y otras creaciones léxicas surgidas a raíz del contacto entre la cocina española e ingredientes y platos procedentes de otros países y culturas.

Para ello, se ha seguido la metodología elaborada por el grupo de investigación El léxico del español en su historia (LEHist) de la Universidad de Verona, que consiste en realizar primero un acercamiento a la estructura de la obra, analizando los elementos de los que se compone, incluso los paratextuales, con el fin de detectar patrones comunes que nos ayuden a ubicar el volumen en el contexto editorial del que surgió. En segundo lugar, tras una atenta criba, se procede al análisis de una selección de términos procedentes del ámbito de especialidad acotado a través de comprobaciones lexicográficas y de corpus para averiguar su trayectoria en la lengua española. Concretamente, las voces extraídas de Agenda culinaria para 1896 pertenecen al dominio de la gastronomía (nombres de ingredientes o platos) y se caracterizan por ser extranjerismos de origen no transparente. Por lo tanto, a efectos de este estudio, ha sido necesario recurrir a obras lexicográficas de otras lenguas europeas también, con especial atención al francés, debido a la destacada tradición culinaria de este país y al reconocido papel de su idioma en la circulación del saber en Europa en la época moderna.

Con respecto a los recursos lexicográficos del español, se ha empleado el Nuevo tesoro lexicográfico de la lengua española (NTLLE) de la Real Academia Española, el Diccionario crítico etimológico castellano e hispánico de Corominas y Pascual (1980-1991); además, se han tenido en cuenta el Diccionario histórico de la lengua española (1933-1936 y 1960-1996) y el Diccionario histórico de la lengua española (2013-), a pesar de que este recurso lexicográfico está todavía en fase de elaboración. En cuanto a la lexicografía francesa, se han consultado las distintas ediciones del Dictionnaire de l'Académie Française, Le trésor de la langue française, el Dictionnaire de la langue française de Émile Littré (1872-1877), disponibles en línea, así como el Dictionnaire historique de la langue francaise de Alain Rey (2010); el diccionario de referencia para la lengua inglesa ha sido el Oxford English Dictionary en línea. En lo que se refiere a la lengua italiana, se ha usado el Grande dizionario della lingua italiana de Salvatore Battaglia (1961-2004), además de las ediciones en línea del diccionario de la Accademia della Crusca. Los corpus empleados en nuestra investigación son los de la RAE, el Corpus Diacrónico del Español (CORDE), el Corpus de Referencia del Español Actual (CRE $A$ ) y el Corpus del Español del Siglo XXI (CORPES XXI), con especial atención al primero de estos por su perspectiva diacrónica. Finalmente, se ha consultado el Fichero general de la RAE, que ha demostrado su utilidad por lo menos en un caso, además del Corpus del Diccionario histórico de la lengua española $(C D H)$. 


\section{Agenda culinaria para 1896. Presentación de la obra}

El autor de la Agenda culinaria publica la primera edición bajo el seudónimo de Blanco Prieto, que, según el catálogo de la BNE, corresponde a Amancio Peratoner y Almirall, un escritor y traductor probablemente natural de Barcelona, del que se conoce solo la fecha de muerte aproximada, 1908. En esa ciudad se publicó la mayoría de sus obras. Según Díez Fernández fue «uno de los erotógrafos más activos de nuestro siglo XIX» (2010: 302), ya que sus temas favoritos se centraban en la higiene sexual, la prostitución y las enfermedades venéreas. Quizás la tarea más relevante de Peratoner como traductor es la que desarrolló participando en la ambiciosa propuesta cultural que representó la colección «Biblioteca Arte y Letras», dirigida por Josep Yxart, para la que tradujo obras de Félix Clément, Alexandre Dumas padre, Émile Zola. Como acertadamente resume Luisa Cotoner en el título de su estudio (2016), Peratoner fue un «traductor sicalíptico».

El volumen Agenda culinaria para 1896 fue publicado por «uno de los más afamados libreros del momento» (Martínez Martín 2001: 459), Bailly-Baillière. La casa editorial contaba con un amplio surtido de libros extranjeros (2001: 459) y se había especializado en la producción y venta de almanaques, enciclopedias y agendas, además de la de libros científicos. La aparición de la Agenda culinaria fue acompañada por una abundante publicidad en la prensa, como puede comprobarse en los numerosos avisos publicados en periódicos y revistas almacenados en plataformas en la web como Hemeroteca digital, de los que reproducimos a continuación algunos:

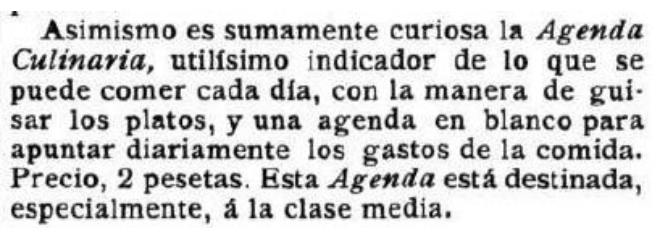

La Iberia, 20 de diciembre de 1895, año XLIII, núm. 14370, p. 3 (Hemeroteca Digita). 
En ambos casos se subraya la absoluta y útil novedad de la presentación con respecto a las usuales y se señala, además, el público interesado («la clase media», «nuestras lectoras»).

La edición de la Agenda culinaria se reiteró probablemente hasta 1936 (según indica el catálogo de la BNE), aunque Peratoner hubiera muerto en 1908. Según los datos de la Biblioteca Digital Hispánica a partir del año siguiente a 1896 el redactor pasó a tomar el seudónimo de Duquesa Laura. En los sitios web de venta de libros antiguos hay algunos ejemplares cuyo formato indica que la macroestructura del volumen permaneció inalterada; esto nos permite afirmar que se trató de un verdadero producto editorial de consumo de la editorial Bailly-Baillière.

El volumen mide $23 \mathrm{~cm}$, un formato manejable, y se compone de algo más de 365 páginas, puesto que unas cuantas están dedicadas a elementos paratextuales, principalmente anuncios de bodegas, tiendas y publicaciones, que se ubican tanto al principio del volumen, entre cubierta y portada, como en las últimas hojas tras el índice. Cada día del año presenta una minuta de 6 o 7 preparaciones para el almuerzo y otras tantas para la comida en la columna a la derecha. De todas estas solamente dos se desarrollan en la explicación en el fondo de la página; la columna de la izquierda está pensada para anotar los gastos efectivos para hacer la compra y preparar los platos del día, permitiendo una gestión eficaz de la economía del hogar.

El formato se repite invariado con excepción del día 15 de cada mes, en el que se presenta un menú para un banquete de 8 o 10 invitados; además, después del último día del mes se inserta una página con los manjares propios, es decir de temporada, del mes siguiente y unos remedios útiles («Procedimiento contra el sudor de las manos» o «Para limpiar los zapatos blancos»). En esta sección en el pie de página se anuncian publicaciones generalmente relacionadas con la familia y el hogar (Higiene, Física y Moral de los niños), o bien con el desarrollo profesional y personal (Guía teóricopráctica para el uso del artista cantante, Guías de Conversación, español-francés, español-inglés, español-francés é inglés).

La sección «manjares propios» sigue el patrón del conocido libro Arte de arreglar comidas, publicado anónimamente en 1869 con el título La gran economía de las familias: arte de arreglar y componer lo sobrante de las comidas de un día para otro [...] por un gastrónomo jubilado, del cual volveremos a hablar en breve. Cabe subrayar que Blanco Prieto en su Agenda culinaria comparte con este libro una estrategia de organización textual, a saber, la presentación de los ingredientes de temporada (carne, pescado, etc.) propios de cada mes. Por cierto, sería oportuno averiguar en trabajos futuros las relaciones textuales y léxicas entre libros «de consumo» como podían ser la Agenda culinaria (recopilada por un redactor que, como se ha visto, no era un cocinero sino un escritor-traductor) y los principales recetarios de la época, por ejemplo, los de Ángel Muro, un conocido cocinero y autor. Por un lado, Blanco Prieto incluye en su agenda algunas recetas del clásico volumen de Francisco Martínez Montiño, publicado en 1611, atribuyéndole su procedencia de forma explícita; por otro, como veremos a continuación, hay coincidencias léxicas que apuntan hacia una cercanía con la obra de Muro. 
Por lo que atañe a la recuperación de recetas de Montiño, cabe observar que la incorporación no es esporádica, sino que se reitera muchas veces a lo largo de la obra, sobre todo en los meses más fríos: Capón relleno, 15 de mayo; Uspot, 4 de junio; Albondiguillas de carpa, 12 de junio; Sopa de perdices, 31 de julio; Perdices asadas con aceite, 5 de septiembre; Lampreas en cecina, 24 de septiembre; Carnero adobado, 1 de octubre; Tortilla con agua y sal, 6 de octubre; Fruta de almendras, 16 de octubre; Sopa de buevos estrellados con leche, 22 de octubre; Colas de carnero con agraz, 28 de octubre; Huevos esponjados, 6 de noviembre; Huevos mejidos, 13 de noviembre; Pierna de carnero estofada, 20 de noviembre; Calabaza frita, 27 de noviembre; Ensalada de zanahorias, 6 de diciembre; Albondiguillas castellanas, 14 de diciembre; Capón á la tudesca, 22 de diciembre; Albondiguillas de pan rallado, 31 de diciembre. Cada una de estas recetas aparece entrecomillada y acompañada de la referencia a la fuente (F. M. Montiño).

Las relaciones intertextuales entre la Agenda culinaria y El practicón de Ángel Muro pueden rastrearse en varios puntos, como la recuperación de la locución adverbial al minuto. En este segundo tratado se lee que: «En algún libro de cocina se llama esta salsa bechamela al minuto, porque se hace en un abrir y cerrar de ojos, pero en general todas las salsas se hacen rápidamente, y podrían llamarse igual» (Muro 1894: 157). Efectivamente Peratoner va aplicando el sintagma a varios casos, de entre los cuales mencionamos Alondras al minuto (9 de febrero), Higado de ternera al minuto (13 de junio), Anguila al minuto (10 de julio).

Se observan, asimismo, algunas coincidencias puntuales en el empleo de determinadas voces léxicas, como albardilla. Se trata de un término no lematizado en los diccionarios según la acepción que indica Muro:

Llamaré así á lo que la cocina francesa llama selle (sillín) de carnero, porque en verdad, de sillín ó de albarda tiene la facha el trozo de carnero que se necesita para los platos que voy á formular á continuación.

La albardilla de carnero, es la parte de la res comprendida entre la pierna y las primeras costillas.

Solamente en las cocinas dispendiosas y dé alto bordo, se puede presentar este plato, y para buen número de comensales, porque resulta uno de los mayores trozos de carne que se sirven en una mesa. En las demás casas, por lo general, aún en las familias ricas y acomodadas, no es condimento más que la mitad de la albarda, y bien deshuesada de antemano.

Esta parte del carnero no es ventajosa, porque tiene mucho hueso y poca carne. (Muro 1894: 362).

Sin embargo, en la Agenda culinaria se reitera muchas veces, como puede comprobarse en recetas como Albardilla de carnero con albondiguillas (27 de abril), Albardilla de carnero asada (23 de junio), Albardilla de carnero á la inglesa (20 de diciembre). ${ }^{1}$

\footnotetext{
1 En el ámbito culinario, el Diccionario histórico de 1933 acoge en su microestructura la acepción de «Lonja de tocino gordo que se pone por encima a las aves para asarlas» y Autoridades recoge, en cambio, la siguiente: «Es tambien una composición de cosas comestibles, que se hace con huevos, harína, dulce, \&c. con que se bañan, rebozan ò envuelven pies y lénguas de puerco, y de otros animales, despojos de aves, y hojas de diferentes hierbas, y otras cosas para comer. Es voz del uso familian»..
} 
En todo caso, conviene destacar el papel que desempeñaron obras como esta en el intento de nacionalización culinaria que tuvo lugar, no solo en España sino en buena parte de Europa, a finales del siglo XIX y principios del XX por parte de quienes - casi en su totalidad varones, con la excepción de Emilia Pardo Bazán (Burdiel 2021) — trataron de fraguar una identidad española en el ámbito de la cocina (Torres Martínez 2021). A este propósito, puede observarse que la Agenda culinaria abarca titulaciones extraídas de un amplio abanico de procedencias, según se comprueba en ejemplos como á la francesa, á la italiana, á la alemana, á la portuguesa, á la holandesa, que se mezclan con una alta frecuencia de recetas de origen americano, como á la matancera, á la cubana, á la mexicana, á la babanera, á la criolla. Naturalmente es una época en la que la cocina americana se percibe como completamente familiar en la metrópoli. Así pues, el poder compartir un listado de recetas comunes a toda España, aunque algunas se hubieran tomado de otras culturas, debió de contribuir a la fragua de esa identidad.

\section{Estudio histórico de una muestra de extranjerismos gastronómicos}

Desde el punto de vista lingüístico, en la Agenda culinaria se puede apreciar la vinculación de la gastronomía española con la de otros países, hecho que se manifiesta no solo en el nombre de algunas recetas (e.g. minestrone á la milanesa), sino también en la presencia en el texto de numerosos galicismos e italianismos, tanto adaptados como en su grafía original, en este último caso con o sin resalte gráfico. Citamos como ejemplos del francés galantina, fricandó, á la maître d'hotel (este último en cursiva en el texto), macarrones, mille-fanti y minestrone (los dos últimos entrecomillados en el texto) del italiano, arrow-root del inglés, un extranjerismo que correspondería a la forma moderna arruruz, lematizada en la edición de 1994 del DRAE. A efecto de este artículo, se ha realizado un vaciado manual de los términos de especial interés en cuanto a su aportación al estudio histórico del léxico.

Concretamente, nos hemos centrado en las lexías kari, uspot y pilaw. Kari aparece en una receta del día 21 de mayo:

Bacalao con arroz blanco

Cocer en agua una porción de trozos de bacalao previamente desalado.

Rehogar en manteca un par de cebollas picadas.

Espolvorearlas con una cucharada de harina y otra de kari, y á los dos minutos mojar poco á poco la mezcla, meneándola, para obtener una salsa clara. [...]

Ahora bien, una búsqueda realizada en NTLLE nos ha devuelto solo un resultado, a saber, el diccionario de Zerolo (1895), que lematiza kari con el significado de «Especia que se produce en América y que sirve para hacer salsas muy fuertes». En CORDE se recogen 9 ocurrencias de kari, todas sacadas de una misma obra: El Practicón. Tratado completo de cocina del ya mencionado Muro que, según CORDE, se publicó en 1891 y de la que pudimos hojear la versión digitalizada de la BNE (5a edición de 1894). Es de especial relevancia uno de los contextos que recoge CORDE: 
He sacado a relucir el kari antes de tiempo, y tengo que decir lo que es y cómo se hace, pues es plato muy conocido en España y muy en uso entre la gente que ha estado en Filipinas, por ser manjar de allá. (Muro 1982 [1891-1894]: 105)

Desde luego, contrariamente a la opinión de Muro, no parece ser un plato demasiado conocido, puesto que las referencias a autores que mencionen este ingrediente son muy escasas; en efecto, además de en El Practicón, kari se lematiza en el Diccionario general de cocina de 1892 de este mismo autor, en el que se ofrece también la receta para su preparación:

En las buenas tiendas de comestibles se vende el kari en polvo molido; pero el que quiera fabricarlo por su cuenta y riesgo, que lea esta fórmula que al objeto traduzco del Indian’s Cook: «El polvo ó harina de kari debe componerse de cuatro onzas de pimiento rabioso, que es un pimientilla, del tamaño de una aceituna, que se cría en los trópicos, mucho más fuerte y picante que nuestra guindilla y que la pimienta de Cayena, tres onzas de terra merita de la India, media onza de pimienta negra, un polvo de nuez moscada y un escrúpulo de jengibre. Se convierte todo esto en polvo fino, moliéndolo bien en mortero de metal con mano de mármol». (Muro 1892: vol. 2, s.v. kari)

El Fichero general de la RAE confirma el uso de este término por parte de Muro con dos ejemplos de El Practicón y de Diccionario general de cocina; además, se guarda una cédula que reproduce un ejemplo extraído de Arte de arreglar comidas de 1869: «Es una especie de pimienta roja en polvo que se vende en las tiendas de ultramarinos».

El Corpus del Diccionario histórico de la lengua española, por su parte, registra las mismas 9 ocurrencias que aparecen en CORDE, todas ellas procedentes de El Practicón. Tratado completo de cocina de Muro. ${ }^{2}$

En la lexicografía francesa la voz cari se lematiza en el Dictionnaire de la langue française de Émile Littré (1872-1877), con el significado de «Poudre de cari, assaisonnement composé de piment, de curcuma et d'autres épices pulvérisées» y la acepción derivada «Volaille ou tout autre mets préparé avec ce condiment. Manger un cari». La entrada se registra también en la $8^{a}$ edición del Dictionnaire de l'Académie Française (1932), «Assaisonnement d'épices pulvérisées. On écrit aussi KARI». El Trésor de la langue française proporciona dos variantes gráficas más, a saber, carry y curry, y deja constancia de un ingrediente más en la preparación de este polvo, el azafrán: «Condiment indien composé de piment, safran, poivre et autres épices pulvérisées. Poudre de carry». Merece la pena destacar que, según el Trésor de la langue française, la voz cari se atestigua en el léxico francés en su variante caril a partir de 1602 y presenta cierta inestabilidad gráfica (cariil en 1610, cary en 1688, carri en 1708). El Dictionnaire historique de la langue française de Alain Rey (2010) también lematiza cari con las variantes carry y curry, rectificando la grafía de 1610 en carril y aclarando que «[]]a forme curry, qui l'emporte au $\mathrm{XX}^{\mathrm{e}}$ s. et élimine les variantes anciennes, est empruntée à l'anglais curry de même origine $[\ldots]$...

2 La coincidencia de resultados entre $C D H$ y CORDE se da también en la búsqueda de las concordancias del término uspot ya que el CORDE, como sabemos, es una de las herramientas que ha servido para alimentar el CDH. 
El Oxford English Dictionary (s.v. curry) ratifica que kari es una voz del tamil que pasó al portugués con la forma caril, al inglés con la forma carriil atestiguada en 1598 y sucesivas variantes, y al francés con las formas tempranas que acabamos de comentar. El periplo de este término conduce a la incorporación del anglicismo curry en el Diccionario de la lengua española con remisión a curri y etimología del tamil kari.

Otro término de interés en el que queremos profundizar es uspot, un plato de carne propuesto para el 4 de junio. Se trata de una receta tomada del conocido libro de Francisco Martínez Montiño Arte de cozina, pasteleria, bizcocheria y conserueria (1611), que Blanco Prieto reproduce citando el texto entre comillas y con su referencia entre paréntesis. La palabra uspot nunca se ha lematizado en los diccionarios académicos, tan solo se recoge una anotación en una cédula del Fichero general, donde se lee «Uspot (1). Es un plato de jarretes de ternera», con referencia al tratado del célebre cocinero de Felipe II. En CORDE, sin embargo, se ha podido encontrar una mención de uspot procedente del recetario de Muro: «No hay duda que [sic] el nombre Hochepot está tomado del Uspot de la cocina antigua española, pues el célebre Montiño da la receta que copio a continuación con su misma ortografía, aunque no se aplique al rabo de vaca» (Muro 1982 [1891-1894]: 166-167).

A partir de esta información, se ha decidido consultar los recursos disponibles de la lexicografía francesa. La búsqueda en el Dictionnaire de l'Académie française ha devuelto un resultado a partir de la cuarta edición de 1762 («HOCHEPOT. s.m. Espèce de ragoût fait de boeuf haché, \& cuit sans eau dans un pot avec des marrons. Un bon hochepot»), que se repite con mínimas ampliaciones en las ediciones quinta de 1798, sexta de 1835 y octava de 1932. Justamente la sexta edición del DAF (1835) —HOCHEPOT s. m. (H s'aspire.) T. de Cuisine. Espèce de ragoût fait de boeuf haché, et cuit sans eau dans un pot, avec des marrons, des navets et autres assaisonnements. Un bon hochepot.»— debió de servir como modelo a Émile Littré para su entrada del Dictionnaire de la langue française, que resulta ser en gran medida idéntica. Según este autor, el término se remonta al siglo XIV («Hochepot de volaille») y su circulación es atestada en varios idiomas europeos: «Hocher 1, et pot; wallon, hose-pot, bouillie; angl. hodge-podge, hotch-potch, de l'anc. flam. hutspot; dialecte d'Aix-la-Chapelle, hoetschpott; holland. hutspot, du flamand hutsen, secouer, et pot». El Trésor de la langue française informatisé, por su parte, confirma la etimología a partir de Hocher, o sea, seconer (con el significado de 'sacudir'), sin embargo, ofrece una primera atestiguación que se remonta a principios del s. XIII. A su vez, el diccionario de Alain Rey (2010), confirma 1200 como probable fecha de la primera atestiguación y la etimología que acabamos de ilustrar: «HOCHEPOT [...] designe un ragoût de bœuf et de volaille (on remue le pot pour que le contenu n'attache pas). Il ne s'emploit plus que dans des usages régionaux» (s.v. hocher 2).

En cuanto a la lexicografía de la lengua inglesa, el Oxford English Dictionary (s.v. hotchpot) confirma la etimología de la voz hasta aquí esbozada: «Anglo-Norman and Middle French bochepot (French bochepot) dish containing a mixture of many ingredients, especially kind of stew made with minced beef or goose and various vegetables (c1214 in Old French)». Estas pesquisas, por un lado, parecen confirmar la suposición formulada por Muro, esto es, cierta correspondencia entre bochepot y uspot, 
aunque el ingrediente principal del plato francés es la carne de ternera en trozos, posiblemente con aves, mientras que la receta de Montiño se prepara con jarretes de ternera; por otro lado, nos hacen suponer que la derivación de un idioma a otro se debió de producir en dirección contraria a la que postuló él, esto es, del francés al español.

Finalmente, el último término de interés que quisiéramos examinar es pilaw, un plato de arroz propuesto para el 2 de noviembre («Pilaw» á la turca). A pesar de su apreciable frecuencia de uso en la actualidad, pilaf - la grafía hoy mayoritaria, como confirman resultados proporcionados por las búsquedas realizadas en CREA y CORPES XXI - entró en el acervo del léxico recogido por la Academia solamente en la última edición del diccionario, es decir, la de 2014. Sin embargo, el término se registró bajo la forma pilau en algunos diccionarios no académicos, como el de Terreros (1786 [1767]) y, posteriormente, los de Domínguez (1853) y Zerolo (1895), como documentan también los recientes estudios de Torres Martínez (2021: 211). La variante gráfica pilau es también la que se recoge en CORDE en dos ocurrencias, ambas procedentes del El Practicón de Muro y ambas compartidas con el $C D H$.

En cuanto a la recepción de este plato por el público español, señalamos un fragmento de un artículo publicado diez años antes de que viera la luz la Agenda culinaria de Blanco Prieto: «un día á la semana le dan carne de carnero, y otro día pilaw, comistrajo compuesto de arroz y manteca de Siberia, muy estimada de los turcos». (La ilustración artística: periódico semanal de literatura, artes y ciencias, vol. 5, núm. 250,11/10/1886). En la cita, que se refiere a las apuradas condiciones de vida de los soldados turcos, se inserta la voz pilaw acompañada de una aposición explicativa (y despreciativa) dirigida al lector de la época, probablemente todavía no muy ducho en este tipo de preparaciones exóticas. Pocos años después, en cambio, la Agenda culinaria de Peratoner lo considera apto para una cena.

En cuanto a la etimología de pilaf, el DLE mismo sugiere la procedencia del francés de esta voz, lengua mediadora entre el turco y la difusión en español. En efecto, el Trésor de la langue française informatisé coincide en señalar que es un préstamo del turco pilâv, y este del persa pilaw. Además, este recurso lexicográfico indica mediados del siglo XVII para la introducción en francés de este orientalismo con la grafía pilau y señala para este idioma las mismas oscilaciones gráficas que ya se han detectado en español (pilaw, pilavv). Desde luego, la forma pilau es la que elige el Dictionnaire de l'Académie Française en las ediciones cuarta (1762), quinta (1798) y sexta (1835) para su definición, «Riz cuit avec du beurre, ou de la graisse \& de la viande. Le pilau est la nourriture ordinaire dans le Levant», así como Émile Littré en su diccionario de 1872-1877. En cambio, los redactores de la octava edición del $D A F$ (1932) se decantan por la más moderna variante pilaf y aprovechan también la ocasión para actualizar la definición con una marca diatécnica «T. de Cuisine. Riz cuit avec des morceaux de viande, de volaille ou de poisson et très épicé. Pilaf de poulet. Pilaf de homard. On dit aussi Pilau». Nótese que ha desaparecido la indicación de la procedencia geográfica del plato, probablemente por haber quedado inadecuada en el primer tercio del siglo XX. Alain Rey, en su diccionario histórico, confirma la oscilación de las formas gráficas pilau (1654), pilaw (1680), pilao (finales del siglo XVIII) y fecha la fijación de la forma moderna, pilaf, en 1833. 
En lo que se refiere a la lexicografía inglesa, el Oxford English Dictionary (s.v pilau) coincide en señalar la gran variación gráfica de esta voz, cuya primera atestiguación se remonta a 1609: «The most common [Turkish] dish is Pilaw... made of Rise, and small morsels of Mutton boyled therein»; sin embargo, el OED amplía el abanico de posibilidades en cuanto a su etimología, pues añade la información que copiamos a continuación: «Probably of multiple origins. Partly a borrowing from Persian. Partly a borrowing from Hindi. Probably also partly a borrowing from French. Probably also partly a borrowing from Italian. Etymons: Persian pulaw; Hindi pulāv; French pilau; Italian pilao». En el mismo artículo lexicográfico, se alude a la fecha de 1542 en relación con el italiano.

Efectivamente, según hemos podido comprobar en el diccionario de Battaglia (1961-2004), la atestiguación más antigua de la voz pilaf en la lengua italiana se encuentra en Relazione di Persia, una obra publicada en 1542 por Michele Membré: «Il viver delli Sofiani... è questo: risi, bogra, cavurman, gaglia pilaf, cavurma pilaf, galia, turchisim, saripilaf, capap giachia, chiorvan; queste sono le manestre loro, tutte con risi cioè corno dir macaroni». Este ejemplo, por lo tanto, sería el primer uso registrado de nuestro término en una lengua europea. En este mismo diccionario, se deja constancia de la oscilación gráfica que afecta a esta vOz en italiano también, puesto que se lematiza pilà con remisión a pilao (que a su vez admite la variante pilau), además de pilâf (con variante pilàw), pilau con remisión a pilao y pilàw con remisión a pilaf.

\section{Conclusiones}

El libro de recetas Agenda culinaria de 1896 de Blanco Prieto, seudónimo de Amancio Peratoner y Almirall, es un producto editorial de consumo que se dirige a un público mayoritariamente femenino de clase media. Se trata de una publicación concebida desde su diseño y consecuente maquetación para responder a una finalidad práctica, la de convertirse en un auxilio al ama de casa a la hora de gestionar todos los aspectos relacionados con la cocina, de la ideación de menús de temporada a la elaboración de los platos, pasando por la administración de los gastos correspondientes.

El estudio ha puesto de relieve la interconexión entre Agenda culinaria de 1896 y otras obras contemporáneas, en particular dos libros del cocinero Ángel Muro - El Practicón, originariamente publicado en 1891, y el Diccionario general de cocina de 1892 - además del anónimo La gran economía de las familias de 1869. No solo la Agenda culinaria de 1896 se inserta en un género textual muy popular a finales de XIX, sino que los puntos en común y la cercanía léxica entre el recetario de Blanco Prieto y las publicaciones aludidas nos hacen pensar que se pueden haber producido fenómenos de intertextualidad y refundición de parte de ellas.

En cuanto al estudio histórico del léxico, en este artículo se ha analizado la trayectoria lexicográfica de tres términos que nos ofrecen una muestra de la circulación europea e internacional del léxico de la comida y la gastronomía. 
El primero de estos, kari, se configura como un término marcado por su doble entrada en la lengua española: en efecto, según las comprobaciones lexicográficas y de corpus que hemos realizado, esta vOZ de origen tamil se atestigua en las lenguas europeas entre finales del siglo XVI y principios del XVII con cierta oscilación gráfica; aparece en español probablemente en la segunda mitad del siglo XIX como préstamo efímero y desaparece al terminar el siglo decimonónico. Sin embargo, a partir del nuevo milenio, vuelve a incorporarse a la lengua española (curri) como préstamo adaptado a partir del inglés curry: esta voz, desde luego, es el resultado de la evolución diacrónica de la antigua forma carriil que nunca desapareció de la lengua anglosajona, sino que siguió adaptándose hasta llegar a la forma conocida en la actualidad.

El caso de uspot, en cambio, es el de un préstamo efímero del francés hochepot, que se registra en la lengua española gracias a Arte de cozina, pasteleria, vižcocheria y conserueria de Francisco Martínez Montiño; a pesar de las cuantiosas reediciones desde la prínceps de 1611, esta voz no parece haberse propagado a otros recetarios españoles, con la excepción de las obras de Ángel Muro, retomadas, como se ha demostrado, por Amancio Peratoner i Almirall.

El último ejemplo estudiado, pilaw, nos ha permitido trazar la evolución gráfica y diacrónica de esta voz en cuatro lenguas europeas a lo largo de cinco siglos, con especial atención a su etimología. En relación con esta, se ha podido comprobar que la primera atestiguación de este orientalismo se remonta a la primera mitad del siglo XVI en una obra redactada en italiano (1542), adelantando en un siglo la primera aparición en una obra francesa (1654) y en medio siglo la primera ocurrencia en inglés (1609).

En cuanto a las potenciales líneas de investigación futuras inauguradas por este primer examen de la Agenda culinaria de 1896, cabe destacar, por un lado, la posibilidad de comprobar las posibles intersecciones y confluencias - $\mathrm{O}$ hasta refundiciones - con los numerosos recetarios publicados a finales del siglo XIX, de los que solo hemos ofrecido una muestra y, por otro, la utilidad de ampliar el estudio histórico de voces especializadas para averiguar la propagación de términos de una lengua a otra como consecuencia de la circulación de las ideas en el espacio europeo. 
Francesca Dalle Pezze \& Elisa Sartor. Agenda culinaria para 1896 de Blanco Prieto. Un estudio histórico del léxico de la gastronomía

\section{Bibliografía}

\section{Fuentes primarias y bibliografía consultada}

Anón. (1869) La gran economía de las familias: arte de arreglar y componer lo sobrante de las comidas de un día para otro [...] por un gastrónomo jubilado, Madrid, López Vizcaino.

Blanco Prieto, A. (1896) Agenda culinaria para 1896. Libro de la compra. Con minutas y recetas para cada uno de los días del año, Madrid, Bailly-Balliere é Hijos.

Burdiel, I. (2021) Emilia Pardo Bazán, Barcelona, Penguin Random House [e-book].

Cotoner, L. (2016) «Amancio Peratoner i Almirall, un traductor sicalíptico», en F. Lafarga / L. Pegenaute (eds.), Autores traductores en la España del siglo XIX, Kassel, Reichenberger, pp. 415-430.

Diccionario Histórico de la Traducción en España, [en línea] < http://phte.upf.edu/dhte/> [01/09/2021].

Martínez Martín, J. A. (2001) «La circulación de libros y la socialización de la lectura. Nuevos públicos y nuevas prácticas», en J. A. Martínez Martín (ed.), Historia de la edición en España 18361936, Madrid, Marcial Pons, pp. 455-472.

Martínez Montiño, F. (1611) Arte de cozina, pasteleria, viz̧cocheria y conserueria, Madrid, Luis Sánchez.

Muro, Á. (1892) Diccionario general de cocina, Madrid, José María Faquineto, 2 vols.

Muro, Á. (1894) El Practicón. Tratado completo de cocina al alcance de todos y aprovechamiento de sobras, Madrid, Librería de Miguel Guijarro, Editor.

Torres Martínez, M. (2021) «Recetas y léxico culinario en prensa española del siglo XIX», Boletín de Filología, t. LVI, n. 1, pp. 203-233.

\section{Recursos lexicográficos y bases de datos}

Accademia della Crusca $(1612,1623,1691,1729-1738,1923)$ Vocabolario degli Accademici della Crusca [en línea] <http://www.lessicografia.it> [24/10/2021].

Battaglia, S. (1961-2004) Grande dizionario della lingua italiana, Torino, UTET [en línea] <http:// www.gdli.it.> [24/10/2021].

Biblioteca Nacional de España Biblioteca Digital Hispánica [en línea] <http://www.bne.es/es/ Catalogos/BibliotecaDigitalHispanica/Inicio/index.html> [01/09/2021].

Biblioteca Nacional de España Hemeroteca digital [en línea] < http://hemerotecadigital.bne.es/index. $v m>[01 / 09 / 2021]$. 
Francesca Dalle Pezze \& Elisa Sartor. Agenda culinaria para 1896 de Blanco Prieto. Un estudio histórico del léxico de la gastronomía

Corominas, J. / Pascual, J. A. (1980-1991) Diccionario crítico etimológico castellano e bispánico, Madrid, Gredos.

Ministerio de Cultura y Deporte Biblioteca virtual de prensa histórica [en línea] <https://prensahistorica. mcu.es/es/inicio/inicio.do> [01/09/2021].

Dictionnaire de l'Académie Française (1694, 1762, 1798, 1835, 1932-5) [en línea] <artfl-project.uchicago. edu/node/17/> [01/09/2021].

Littré, É. (1872-1877) Dictionnaire de la langue française [en línea] < https://www.littre.org/> [01/09/2021].

Oxford English Dictionary Online (OED), [en línea] <http://www.oed.com/> [01/09/2021].

Real Academia Española y Fundación Rafael Lapesa Fichero general [en línea] <http://www.frl.es/ Paginas/FicherolexicoRAE.aspx> [01/09/2021].

Real Academia Española Corpus Diacrónico del Español (CORDE) [en línea] < http://corpus.rae.es / cordenet.html> [01/09/2021].

Real Academia Española CORPES XXI. Corpus del Español del Siglo XXI [en línea] < https://www. rae.es/banco-de-datos/corpes-xxi> [01/09/2021].

Real Academia Española (2013) Corpus del Diccionario bistórico de la lengua española (CDH) [en línea] $<$ https://apps.rae.es/CNDHE > [01/09/2021].

Real Academia Española CREA. Corpus de Referencia del Español Actual [en línea] <https://www.rae. es/banco-de-datos/crea> [01/09/2021].

Real Academia Española Diccionario histórico de la lengua española (DHLE) [en línea] <https://www. rae.es/dhle/> [01/09/2021].

Real Academia Española Nuevo tesoro lexicográfico de la lengua española (NTLLE) [en línea] < http:/ /www. rae.es/recursos/diccionarios/diccionarios-anteriores-1726-1992/nuevo-tesoro-lexicografico> [01/09/2021].

Rey, A. (dir.) (2010) Dictionnaire historique de la langue française, Paris, Le Robert, 3.a ed.

Trésor de la langue française [en línea] <atilf.atilf.fr/> [01/09/2021].

SCRIPTA, Revista internacional de literatura i cultura medieval i moderna, núm. 18 / desembre 2021 / pp. 115-127 ISSN: 2340-4841· doi:10.7203/SCRIPTA.18.22767 